\title{
THEORETICAL STUDIES OF COLLECTIVE ION ACCELERATION IN A LINEAR BEAM-EVACUATED DRIFT TUBE SYSTEM ${ }^{+}$
}

J. M. Grossmann, I. Mayergoyz, and C. D. Striffler

University of Maryland, College Park, Maryland 20742

Abstract. This paper deals with recent theoretical studies and ideas on Collective Ion Acceleration (CIA) in systems in which a linear intense electron beam is injected into an evacuated drift tube. The source of ions in these systems is the result of electrical breakdown of a dielectric insert in the anode or ionization of a localized gas cloud near the beam entrance. A phenomena basic to these CIA experiments is the virtual cathode formation process. We present numerical studies of this process using a new particle simulation code that features a non-uniform mesh. Also, we present a model of the entire CIA process, delineating the role of the virtual cathode.

\section{Introduction}

A schematic of the CIA system under study at the University of Maryland is shown in Figure 1. An in-

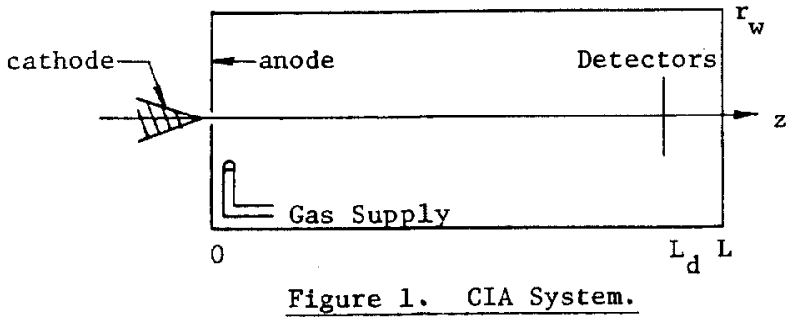

tense relativistic electron beam is injected into a cylindrical drift tube with a maximum current that is above the limiting current of the system. Normal beam parameters in the experiments are; peak voltage $\mathrm{V}_{\mathrm{O}}=1 \mathrm{MV}$, peak current 40KA, pulse width 30 nsec. The supply of ions for acceleration is furnished by a gas puff value located ncar the beam entrance as shown in Figure 1. Typical dimensions are; anode hole diameter $2 \mathrm{~cm}$, cylindrical tube radius and length, $12.5 \mathrm{~cm}$ and $1 \mathrm{~m}$, respectively, detector location $\mathrm{L}_{\mathrm{D}}-60 \mathrm{~cm}$. Experiments on this sort of system were initiated by Luce 1 and have been followed by similar experiments at Maryland $2-5$ using dielectric inserts as well as a localized gas cloud.

The main observations in the experiments at Maryland are: 1. No ion acceleration is observed unless the electron current is above the limiting current. one such limiting current is that due to a space-charge limit; this well known result is $\mathrm{I}_{\mathrm{L}}=17\left(\gamma_{\mathrm{o}}^{2 / 3}-1\right)^{3 / 2}$ / $\left[1+2\right.$ ln $\left.r_{w} / r_{b}\right] \mathrm{KA}$. With our parameters this limiting current is between 1 and 3KA. $\underline{2}$. The second observation is that the amount of electron charge observed at the detector is substantially larger (5-10 times) when CIA is observed versus when it is not. $\underline{3}$. The ion enerty distribution measured at the detector possesses a broad peak in the energy region (1-3) eV and an exponential-like tail that terminates in the region $(5-10)$ $\mathrm{eV}_{\mathrm{O}}$. The number of ions at the extreme high energy end of the spectrum is down by a factor of about $10^{5}$ from the number in the main body of the spectrum. 4. Another observation is that the acceleration process occurs in a relatively short distance from the anode. 5. Finally, the ions in the high energy tail arrive at the detector almost simultaneously with the arrival of the electron pulse and this occurs at a time near the end of the diode pulse, i.e., $30 \mathrm{nsec}$ after the system is fired. Lower energy ions follow at a later time.

Fhis work is supported by NSF, AFOSR and the University of Maryland Computer Sclence Center.

\section{Virtual Cathode Dynamics ${ }^{*}$}

A complete understanding of the mechanfsm for CIA has long allured and eluded researchers in the field. A phenomena basic to these CIA experiments is the virtual cathode (V.C.) formation process which occurs when the injected current is above the limiting current of the system. Our numerical investigation of this process has yielded many results in agreement with other simulations. However, we would like to improve the resolution of the region near the v.c. using a non-uniform mesh potential solver. We introduce several geometrical simplifications into our analysis; namely, the beam motion is purely axial; the beam is thin relative to the drift tube radius, the charge density is radially uniform, and finally, axial symmetry is imposed. A potential solver tailored specifically to these features of the system has been developed. It is a semianalytic method which reduces the problem to an ODE via the Galerkin procedure.

On a unfform mesh with radially uniform charge density, it is possible to solve poisson's equation on axis with an asymptotic operations count of $0(n l n n)$, which increases to $0\left(\mathrm{n}^{2}\right)$ on a non-uniform mesh. The freedom of a non-uniform mesh allows us to position the grid points in order to obtain better resolution of the virtual cathode.

The Poisson Equation for the potential $\phi(r, z)$ in cylindrical coordinates with axial symmetry is

$\left[\frac{\partial^{2}}{\partial z^{2}}+L_{r}\right\} \phi(r, z)=-\rho(r, z) / \varepsilon_{0}$,

where the operator $L_{r} \equiv \frac{1}{r} \frac{\partial}{\partial r} \frac{r \cdot}{\partial r}$, and $\rho(r, z)$ is the beam charge density. After the Galerkin method in the variable $z$ is applied to Equation (1), we arrive at a system of equations;

$$
\begin{gathered}
\int \sum_{n=1}^{N} \Lambda_{n}(z) L_{r} \phi_{n}(r) \Lambda_{M}(z)+\sum_{n=1}^{N} \rho_{n} \frac{\Lambda_{n}}{\varepsilon_{0}}(z) \Lambda_{M}(z)- \\
\left.\sum_{n=1}^{N} \phi_{n}(r) \frac{d \Lambda_{n}}{d z} \frac{d \Lambda_{M}(z)}{d z}\right\} d z=0, M=1, \ldots, N,
\end{gathered}
$$

where $\Lambda_{\mathrm{n}}$ 's are a set of known basts functions defined on the interval $[0, L]$, and we have used the following approximations to the functions $\phi(r, z)$ and $\rho(r, z)$

$$
\tilde{\phi}(r, z)=\sum_{n=1}^{N} \phi_{n}(r) \Lambda_{n}(z), \tilde{\rho}(r, z)=\sum_{n=1}^{N} \rho_{n}(r) \Lambda_{n}(z) .
$$

Equations (2) can be written as the matrix equation

$$
\sum_{n=1}^{N} B_{M n} L_{r} \phi_{n}(r)+\sum_{n=1}^{N} A_{M n} \phi_{n}(r)+\sum_{n=1}^{N} B_{M n} \frac{\rho_{n}(r)}{\varepsilon_{0}}=0 \text {, }
$$

$\mathrm{M}=1, \ldots \mathrm{N}$,

where by comparing Equations (2) and (4), the matrices A and $B$ can be obtained easily.

The problem of diagonalizing this system is equiyalent to the generalized eigenvalue problem: $\overrightarrow{A x}=\lambda \vec{B} \vec{x}$. With our cholce of basis functions, $A$ and $B$ become symmetric, and $B$ positive definite. These are the 
conditions guaranteeing the existence of a matrix $R$ such that $\mathrm{R}_{\mathrm{TR}}=\Delta$, a diagonal matrix, and $\mathrm{R}_{\mathrm{BR}}=\mathrm{I}$, the identity matrix. ( $\mathrm{R}^{\mathrm{T}}$ is the transpose of R.) Our Equation (4) can be converted with matrix $R$ to a system of modified Bessel Equations;

$\mathrm{L}_{\mathbf{r}} \psi+\Delta \psi=-\mathrm{R}^{\mathrm{T}} \mathrm{B}_{\rho} / \varepsilon_{\mathbf{o}}$,

with $\psi=\mathrm{R}^{-1} \phi$. Equation (5) is a vector equation and each component can be solved using a Green's function formalism. Specifically, the $i^{\text {th }}$ component of $\psi$ becomes

$\psi_{i}=-G_{i}\left(R^{T} B \rho / t_{o}\right)_{i}$,

where

$G_{i}=\frac{1}{\lambda_{i} I_{0}\left(\lambda_{i} r_{w}\right)}\left\{r_{b} K_{0}\left(\lambda_{i} r_{w}\right) I_{1}\left(\lambda_{i} r_{b}\right)+\right.$

$\left.r_{b} I_{0}\left(\lambda_{i} r_{w}\right) k_{1}\left(\lambda_{i} r_{b}\right)-\frac{I_{0}\left(\lambda_{1} r_{w}\right)}{\lambda_{i}}\right\}$

and $r_{w}$ is wall radius, $r_{b}$ the beam radius, and $\lambda_{i}=\sqrt{-d_{i}}$. The numbers $d_{i}$ are the eigenvalues of the generalized eigenvalue problem. The coefficients $G_{i}$ need only be computed once during the program and drawn on again whenever necessary. At any given time step, the greatest effort in computing the potential is spent on the matrix multiplication $\mathrm{R}^{\mathrm{T}} \mathrm{B}$ and subsequent synthesis of $\phi=R \psi$. The most time-consuming part of the entire calculation is the solution of the generalized eigenvalue problem which computes the eigenvalues $d_{i}$ and the transformation matrix $R$. Here again, this part need only be computed once. Our numerical model is shown in Figure 2.

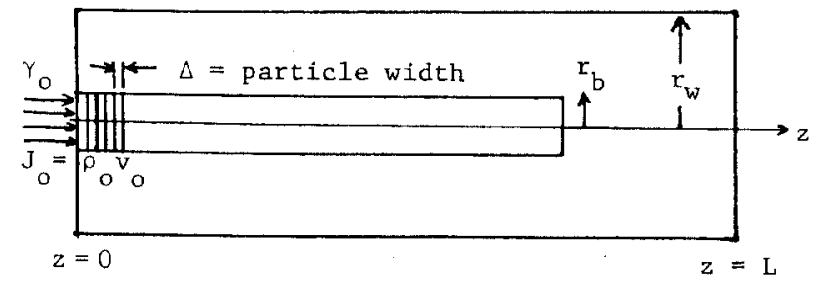

Figure 2. Drift Tube and Beam Model.

A standard area-weighted scheme is employed to compute the charge density from the particle (cloud) positions ${ }^{7}$, and the particles are pushed using a time-centered leap-frog scheme; fields and particle positions being evaluated at whole-time steps, while particle momenta and velocities are found at half-time steps.

Some results from the code's simulation using a current step of $I=10 I_{\tau}$ are shown in Figure 3 . The following parameters are used: $\mathrm{L}=50 \mathrm{~cm}, \mathrm{r}_{\mathrm{w}}=2 \mathrm{~cm}$, $r_{b}=.5 \mathrm{~cm}$ and $\gamma_{0}=2.0\left(v_{0}=.511 \mathrm{MV}\right)$. The grid sizes vary from about $.1 \mathrm{~mm}$. near the anode to $2 \mathrm{~cm}$. near the downstream end wall; and the particle-clouds are $.1 \mathrm{~mm}$ long. The injected beam charge density is approximately 1 coul $/ \mathrm{m}^{3}$, so a value close to 2 coul $1 / \mathrm{m}^{3}$ at the beam entrance, as indicated by Figure $3(a)$, shows that the beam is mustly reflected. Comparisons between our present code and our previous uniform-mesh code ${ }^{7}$ indicate that for the same overall resolution, we can reduce the computer's effort by one quarter. Likewise, the same amount of computer effort can now produce smoother fields. Figures $3(\mathrm{~b})$ and 3 (c) are plots of the corresponding potential and electric field at $.27 \mathrm{~ns}$, well after the V.C. is originally formed. In this particular figurc, the V.C.'s position

*From a dissertation to be submitted to the Graduate School, University of Maryland. is $2 \mathrm{~mm}$. and its depth is $\sim .63 \mathrm{MV}$, or about $1.23 \mathrm{~V}_{0}$. In our simulations the V.C. oscillates between about 2 and $3 \mathrm{~mm}$ in posilion frum the anode and up to about 1.6 $V_{0}$, with frequency on the order of the plasma frequency.
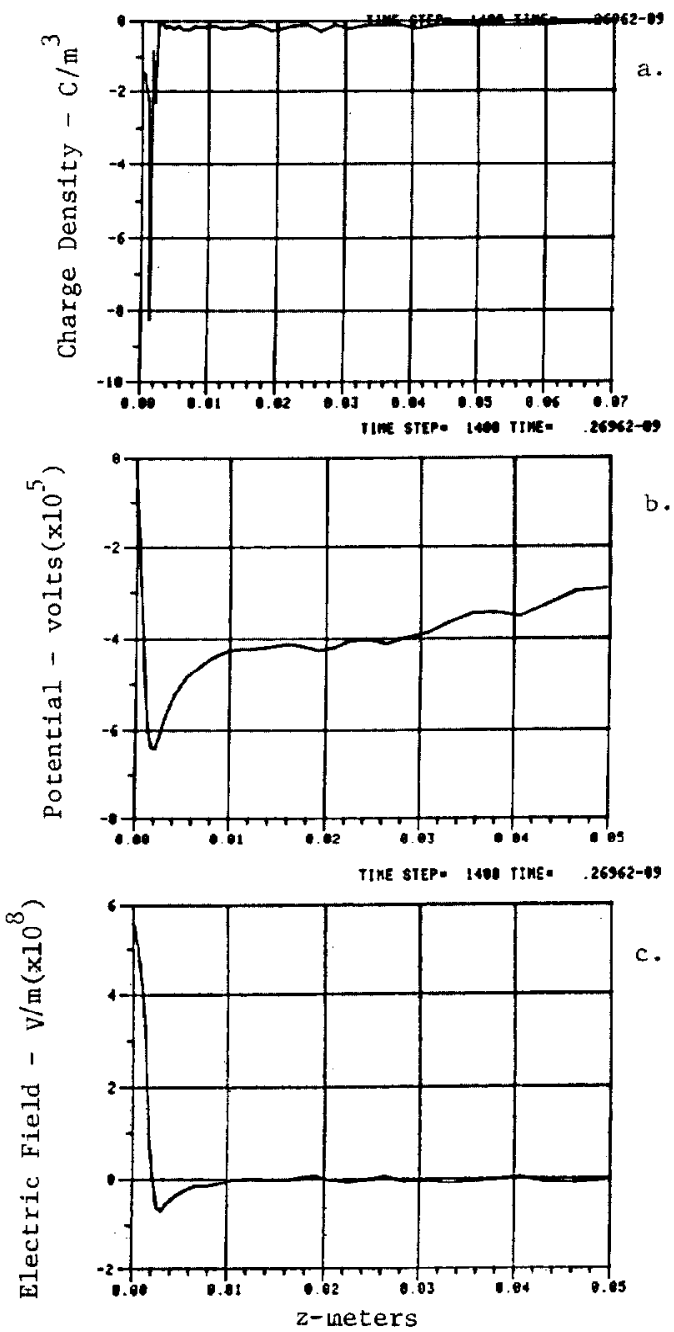

Figure 3. Numerical Results for $I / I_{L}=10, L=.5 m$, $r_{b}=.5 \mathrm{~cm}, r_{w}=2 \mathrm{~cm}, \gamma_{0}=2$. Current Pulse is a unit step.

\section{CIA Mode1}

We now present a model of the CIA process in the system described above, keeping in mind the five stated experimental observations in Section $I$. We break the process down into four stages as depicted in Figure 4. The first stage involves the formation of a V.C. shown in Figure 4(a). This occurs when the beam current is above a limiting value, and the majority of the beam particles are either reflected by the V.C. and/or are scattered radially. In any case a deep potential well is formed near the anode with a depth on the order of $-v_{O}$. The beam density profile is likewise highly peaked in the vicinity of the bottom of the potential well. This stage developes very fast and settles down to some sort of steady state behavior. The concept of V.C. formation is confirmed by experimental observations and further substantiated by the analysis of Section II.

The second stage, shown in Figure $4(\mathrm{~b})$, involves the accumulation of ions in the V.C. region. Due to primary beam ionization of a localized gas cloud, see Figure 1 , the ion-electron pairs are created near the 


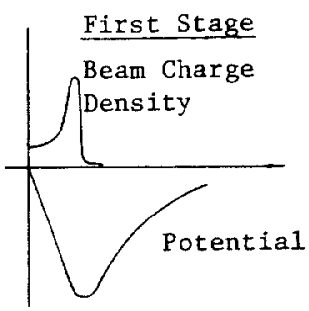

a. Virtual Cathode Formation

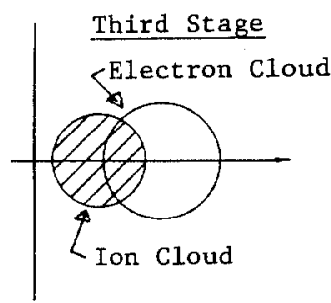

c. Cloud Separation

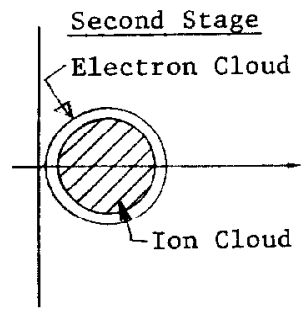

b. Ion Cloud Formation

Fourth Stage

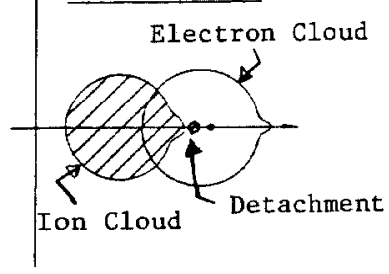

d. Ion Accéleration

\section{Figure 4. CLA Model.}

bottom of the potential well because the beam density is largest in this region and the beam velocity is very low. The secondary electrons created feel a very strong electric fleld and thus are ejected from this region, but the ions are trapped. However, the beam current is sufficiently large that instead of charge neutralization occuring, the V.C. is maintained by the replacement of the ejected secondary electrons by beam electrons. Thus as ionization occurs not only do the number of ions increase but also the number of electrons, in such a way as to maintain a V.C. near the anode region. Thus, a dynamic equilibrium is created between an ion cloud and a beam electron cloud that allows substantially more total beam charge in the system than when no lons are present.

The third stage, cloud separation, shown in Figure 4 (c) occurs because the dynamic equilibrium is unstable. The driving force for separation can be due to the inductive electric field created by the sharply falling beam current. The unstableness has many features similar to the streaming instability and possesses the onset condition that a sufficient number of ions must be present. When cloud separation occurs, a dynamic situation is set up where the potential well depth is deeper than $-v_{O}$ because the total beam charge in the system is greater with the presence of lons. Thus, the resulting local electric fields are also greater than normal.

As a result of separation, a localized group of ions at the edge of the ion cloud experiences intense forces of repulsion due to the bulk of the fon cloud and at the same time attractive forces due to the separated electron cloud. This "special" group of ions located in the region of an extremely intense static electric field are the fortunate group of ions to be accelerated to high energies. This last process is the fourth stage and is depicted in Figure 4(d), where we have attempted to display qualitatively the resulting elongation of the two clouds after separation as well as the detachment of a small select group of ions. Because the small group of ions experiences such strong electric fields, it is possible for them to gain a great kinetic energy in a few centimeters.

In summary, the CIA phenomena is very complicated and in order to understand the actual mechanism of ion acceleration it's expedient to move forward in two directions; 1) to develope qualitative ideas concerning the CIA mechandsm which are consistent with the experimental results; and 2) to verify these ideas by means of detailed numerical simulations.

The authors wish to acknowledge the useful discussions with the other members of the Charged Particle Research Group at the University of Maryland, especial$1 y$, Drs. Destler and Reiser.

\section{$\underline{\text { References }}$}

1. J. S. Luce, Ann. N.Y. Acad. Sci. 20, 336 (1973).

2. G. T. Zorn, H. Kim, C. Boyer, IEEE Trans, NS-22, 999 (1975).

3. C. N. Boyer, W. W. Destler, and H. Kim, IEEE Trans. NS-24, 1625 (1977).

4. W. W. Destler, H. S. Uhm, H. Kim, and M. P. Reiser, J. App1. Phys. 50, 3015 (1979).

5. W. W. Destler, L. E. Floyd, J. T. Cremer, C. R. Parsons, M. Reiser, J. W. Rudmin, IEEE June 1981 issue (this conference).

6. D. J. Sullivan and R. J. Faehl, Bull. APS 24, 1013 (1979).

7. J. M. Grossmann, R. Kulkarni, C. D. Striffler, and R. J. Faehl, Proc. Ninth Conf. on Numerical Simulation of Plasmas, June 30 - May 2, 1980, Northwestern Univ. 